\title{
UNIFORM SUPERCONVERGENCE ANALYSIS OF THE DISCONTINUOUS GALERKIN METHOD FOR A SINGULARLY PERTURBED PROBLEM IN 1-D
}

\author{
ZIQING XIE AND ZHIMIN ZHANG
}

\begin{abstract}
It has been observed from the authors' numerical experiments (2007) that the Local Discontinuous Galerkin (LDG) method converges uniformly under the Shishkin mesh for singularly perturbed two-point boundary problems of the convection-diffusion type. Especially when using a piecewise polynomial space of degree $k$, the LDG solution achieves the optimal convergence rate $k+1$ under the $L^{2}$-norm, and a superconvergence rate $2 k+1$ for the one-sided flux uniformly with respect to the singular perturbation parameter $\epsilon$. In this paper, we investigate the theoretical aspect of this phenomenon under a simplified ODE model. In particular, we establish uniform convergence rates $\sqrt{\epsilon}\left(\frac{\ln N}{N}\right)^{k+1}$ for the $L^{2}$-norm and $\left(\frac{\ln N}{N}\right)^{2 k+1}$ for the one-sided flux inside the boundary layer region. Here $N$ (even) is the number of elements.
\end{abstract}

\section{Introduction AND STATEMENT OF THE MAIN RESUlT}

It is common knowledge in the scientific computing community that discontinuous Galerkin (DG) methods, especially the local discontinuous Galerkin (LDG) method [4, are effective for convection dominated convection-diffusion problems 2]. Numerical experiments revealed some uniform superconvergence phenomena of the LDG method for singularly perturbed problems. It has been observed that (see, e.g., 14]) the LDG method converges uniformly under the Shishkin mesh for singularly perturbed two-point boundary problems of the convection-diffusion type. In particular, the rate of convergence for the one-sided flux was found to be of order $2 k+1$ (comparing with the optimal global rate $k+1$ ), which is uniformly valid with respect to the singular perturbation parameter $\epsilon$. Nevertheless, there is no theoretical justification of this phenomenon so far. In this paper, we investigate the uniform convergence/superconvergence properties of the LDG method under the Shishkin type meshes for a simple one-dimensional model problem on $\Omega=(0,1)$ :

$$
-\epsilon q^{\prime}+b q=f, \quad q(1)=1 .
$$

Received by the editor March 6, 2008.

2000 Mathematics Subject Classification. Primary 65N30, 65N15.

Key words and phrases. Superconvergence, discontinuous Galerkin method, finite element, singularly perturbed problem, convection-diffusion.

The first author's work was supported in part by the Programme for New Century Excellent Talents in University (NCET-06-0712), the National Natural Science Foundation of China (NSFC 10871066, 10571053), and the Excellent Youth Project of Scientific Research Fund of Hunan Provincial Education Department (0513039).

The second author's work was supported in part by the US National Science Foundation grant DMS-0612908. 
To fix the boundary layer at $x=1$, we assume that $b \geq b_{0}>0$. If $f(x)$ is not the fundamental solution $e^{\int_{1}^{x} b / \epsilon}$, the exact solution of (1.1) is

$$
q(x)=q(1) e^{\int_{1}^{x} b / \epsilon}+\int_{x}^{1} \epsilon^{-1} f(t) e^{\int_{t}^{x} b / \epsilon} d t,
$$

which can be decomposed into $q=\bar{q}+q_{\epsilon}$, where

$$
|\bar{q}|_{k+1} \leq C, \quad\left|q_{\epsilon}^{(s)}(x)\right| \leq C \epsilon^{-s} e^{-b_{0}(1-x) / \epsilon} .
$$

Note that when $f \in H^{1}(0,1)$, the solution can be written as

$$
q(x)=\left[q(1)-\frac{f(1)}{b(1)}\right] e^{\int_{1}^{x} b / \epsilon}+\int_{x}^{1} \frac{f^{\prime}(t)}{b(t)} e^{\int_{t}^{x} b / \epsilon} d t .
$$

Our model problem has all the essential properties of the following two-point boundary value problem:

$$
-\epsilon u^{\prime \prime}+b u^{\prime}+c u=f, \quad u(0)=0, \quad u^{\prime}(1)=1 .
$$

Note that when $c=0$, (1.4) can be decomposed into (1.1) and

$$
u^{\prime}-q=0, \quad u(0)=0 .
$$

When both $b$ and $c$ are constants, we may transfer (1.4) into

$$
-\epsilon v^{\prime \prime}+\tilde{b} v^{\prime}=\tilde{f}, \quad \tilde{b}=b-2 \epsilon t, \quad \tilde{f}(x)=e^{-t x} f(x),
$$

by the transformation $u(x)=v(x) e^{t x}$ with $t$ satisfying $-\epsilon t^{2}+b t+c=0$.

The existence of the boundary layer term $q_{\epsilon}$ causes difficulty in the numerical approximation for $\epsilon \ll 1$. It is well known that the standard continuous Galerkin method results in non-physical oscillatory numerical solutions for small $\epsilon$ under quasi-uniform grids. On the other hand, DG methods are able to avoid the oscillation. However, in order to capture the boundary layer behavior, mesh refinement inside the boundary layer is needed. Towards this end, we first select a transition number $\tau \in(0,1 / 2)$, then we partition the interval $(0,1)$ into $N$ (even) subintervals with $N / 2$ of length $H=2(1-\tau) / N$ in $(0,1-\tau)$ and $N / 2$ of length $h=2 \tau / N$ in $(1-\tau, 1)$. With the nodal points being $0=x_{0}<x_{1}<\cdots<x_{N}=1$, we define $\Omega_{j}=\left(x_{j-1}, x_{j}\right), h_{j}=x_{j}-x_{j-1}$, and the DG space as

$$
V_{N}^{\epsilon, k}=\left\{v \in L^{2}(0,1):\left.v\right|_{\Omega_{j}} \in P_{k}\left(\Omega_{j}\right), j=1,2, \ldots, N\right\},
$$

where $\mathcal{P}_{k}$ is the space of polynomials of degree no more than $k$. In this paper, we consider two different mesh refinement strategies by two different selections of the transition number:

$$
\tau=\tau_{\epsilon}=\frac{\epsilon}{b_{0}}(k+1) \ln \frac{1}{\epsilon},
$$

or

$$
\tau=\tau_{N}=\frac{\epsilon}{b_{0}}(2 k+1) \ln N .
$$

The first choice fixes the transition point for a fixed $\epsilon$. It is well known that the boundary layer width is $O\left(\epsilon \ln \epsilon^{-1}\right)$. The second choice is the so-called Shishkin mesh; the transition point changes with each different $N$. 
We use the standard notation $Q_{j}^{+}, Q_{j}^{-},[Q]_{j}=Q_{j}^{+}-Q_{j}^{-}$to introduce the DG scheme for (1.1): Find $Q \in V_{N}^{\epsilon, k}$ with $Q_{N}^{+}=1$ such that for $j=1,2, \ldots, N$,

$$
\int_{\Omega_{j}} Q\left(v^{\prime}+b \epsilon^{-1} v\right)-Q_{j}^{+} v_{j}^{-}+Q_{j-1}^{+} v_{j-1}^{+}=\int_{\Omega_{j}} \epsilon^{-1} f v, \quad \forall v \in V_{N}^{\epsilon, k}
$$

or equivalently, via integration by parts on the above,

$$
\int_{\Omega_{j}}\left(-Q^{\prime}+b \epsilon^{-1} Q\right) v-[Q]_{j} v_{j}^{-}=\int_{\Omega_{j}} \epsilon^{-1} f v, \quad \forall v \in V_{N}^{\epsilon, k} .
$$

Summing up the last $N-n+1$ elements, we obtain for any $v \in V_{N}^{\epsilon, k}$,

$$
\begin{aligned}
a_{n}(Q, v) & =\int_{x_{n-1}}^{1} Q\left(v^{\prime}+b \epsilon^{-1} v\right)+Q_{n-1}^{+} v_{n-1}^{+}-Q_{N}^{+} v_{N}^{-}+\sum_{j=n}^{N-1} Q_{j}^{+}[v]_{j} \\
& =\int_{x_{n-1}}^{1} Q\left(v^{\prime}+b \epsilon^{-1} v\right)+Q_{n-1}^{+} v_{n-1}^{+}-Q_{N}^{+} v_{N}^{+}+\sum_{j=n}^{N-1} Q_{j}^{+}[v]_{j}=\int_{x_{n-1}}^{1} \epsilon^{-1} f v
\end{aligned}
$$

$a_{n}(Q, v)=\int_{x_{n-1}}^{1}\left(-Q^{\prime}+b \epsilon^{-1} Q\right) v-\sum_{j=n}^{N-1}[Q]_{j} v_{j}^{-}=\int_{x_{n-1}}^{1} \epsilon^{-1} f v$.

Here we require $Q_{N}^{-}=Q_{N}^{+}$and $v_{N}^{-}=v_{N}^{+}$according to the boundary condition at $x_{N}=1$. Due to the ODE nature, the solution can be obtained via backward solving.

Remark. The LDG method [4, when applied to the full scale two-point boundary problem (1.4), contains two important steps [2]: 1) transfer the original problem to a first-order ODE system by introducing (1.5); and 2) choose numerical fluxes for $Q$ and $u$ differently (one up-winding and another down-winding) according to the location of the boundary layer.

Although numerical evidence indicates that the LDG method has the same convergence and superconvergence rate for the full scale problem, the theoretical analysis for the ODE model does not carry over to the full scale case when $b$ and $c$ are not constants.

It is worthy to point out that the LDG method, when applied to our simplified ODE model (1.1), is equivalent to the DG time-stepping method introduced in [12, Chapter 14].

Now we are ready to state our main result.

Theorem 1.1. Let $q$ be the solution of (1.1). Assume that $b \geq b_{0}>0$ and $f$ are sufficiently smooth such that the solution $q$ satisfies the regularity (1.3). Let $Q \in V_{N}^{\epsilon, k}$ be the numerical solution of (1.10) (or (1.11)). Then there is a constant $C(k)$, depending on $k$, but independent of $N$ and $\epsilon$, such that

1) If the transition point is given by (1.6), then

$$
\|q-Q\| \leq C(k)\left(\sqrt{\epsilon}\left(\frac{\ln \epsilon^{-1}}{N}\right)^{k}+\frac{1}{N^{k+1}}\right) ;
$$


furthermore, if $q=q_{\epsilon}$ and $x_{n-1} \in[1-\tau, 1)$, then

$$
\begin{gathered}
\|q-Q\| \leq C(k) \sqrt{\epsilon}\left(\frac{\ln \epsilon^{-1}}{N}\right)^{k}, \\
\left|(q-Q)_{n-1}^{+}\right| \leq C(k)\left(\frac{\ln \epsilon^{-1}}{N}\right)^{2 k+1}, \quad n=1,2, \ldots, N .
\end{gathered}
$$

2) If the transition point is given by (1.7), then

$$
\|q-Q\| \leq C(k)\left(\sqrt{\epsilon}\left(\frac{\ln N}{N}\right)^{k}+\frac{1}{N^{k+1}}\right) ;
$$

furthermore, if $q=q_{\epsilon}$ and $x_{n-1} \in[1-\tau, 1)$, then

$$
\begin{gathered}
\|q-Q\| \leq C(k) \sqrt{\epsilon}\left(\frac{\ln N}{N}\right)^{k}, \\
\left|(q-Q)_{n-1}^{+}\right| \leq C(k)\left(\frac{\ln N}{N}\right)^{2 k+1}, \quad n=1,2, \ldots, N .
\end{gathered}
$$

Here we use the simplified notation $\|\cdot\|$ to denote the $L^{2}$-norm on the whole domain $\Omega=(0,1)$. Later on, we will use an index to indicate the $L^{2}$-norm on a subdomain when necessary.

Prior to a formal proof (which will be postponed to the next two sections), we will make some remarks on the literature.

First we compare the LDG with the standard finite element method (under the Shishkin mesh), which has the following error bound (see [15]) for problem (1.4):

$$
\left\|u-u_{N}\right\|_{\epsilon, N} \leq C\left[\frac{1}{N^{k}}+\left(\frac{\ln N}{N}\right)^{k+1}\right] .
$$

Here

$$
\|v\|_{\epsilon, N}^{2}=|v|_{\epsilon, N}^{2}+\|v\|^{2}, \quad|v|_{\epsilon, N}^{2}=\epsilon \sum_{i=1}^{N} h_{i} \sum_{j=1}^{k} w_{j} v^{\prime}\left(x_{i j}\right)^{2},
$$

where $x_{i j}$ are the Gaussian points in element $\left(x_{j-1}, x_{j}\right)$. We see that the LDG does a better job in approximating $q=u^{\prime}$. Note the $\epsilon$ factor in defining the discrete norm $|v|_{\epsilon, N}$.

To the best of our knowledge, (1.13) or (1.15) is the first such kind of theoretical result for DG methods. We would like to emphasize that the central point here is the $\epsilon$ independence. Otherwise, there are many results in the literature which have norms of the solution appearing in the error bounds. Here we list a few.

In the early 1970's, Douglas and Dupont proved that the continuous Galerkin approximation for the two-point boundary value problem

$$
-a u^{\prime \prime}+b u^{\prime}+c u=f, \quad u(0)=0=u(1),
$$

superconverges at all nodal points for $u \in H^{r}$ :

$$
\left|\left(u-u_{h}\right)\left(x_{j}\right)\right| \leq C h^{k+s}\|u\|_{s+1}, \quad s=\min (k, r-1),
$$

compared with the global optimal rate $h^{s+1}$. This result is sharp [5]. Especially when $k=r-1$, the convergent rate is improved from $h^{k+1}$ to $h^{2 k}$. 
Almost at the same time, Wheeler proposed the following flux approximation for the continuous Galerkin approximation:

$$
\Gamma_{j}=x_{j}^{-1}\left[a\left(u_{h}^{\prime}, 1\right)_{\left(0, x_{j}\right)}+\left(b u_{h}^{\prime}+c u_{h}+f, x\right)_{\left(0, x_{j}\right)}\right]
$$

to obtain

$$
\left|\Gamma_{j}-a\left(x_{j}\right) u^{\prime}\left(x_{j}\right)\right| \leq C h^{k+s}\|u\|_{s+1} .
$$

Especially when $k=r-1$, the convergent rate is improved from $h^{k}$ to $h^{2 k}[13$.

With the higher regularity assumption $u \in H^{3 k+2}$, Thomée proved that a DG approximation to the ODE system $u^{\prime}+A u=f$ at nodes converges at rate $h^{2 k+1}$; see [12, Theorem 12.3 (p.189)]. Here, the regularity assumption can be relaxed to $u \in H^{k+1}$ [1, Theorem 7.3.1 (p.248)].

For the DG approximation of a class of transient hyperbolic equations, under locally translation-invariant meshes, Cockburn et al. proved that a simple convolution post-processing improves the rate of convergence from $h^{k+1 / 2}$ to $h^{2 k+1}$ [3].

More recently, Celiker and Cockburn proved that the numerical trace (including flux) of the LDG approximation to problem $-\epsilon u^{\prime \prime}+a u^{\prime}=f$ converges at the rate $h^{2 k+1}$ for $\epsilon=O(1)$ [2].

Of course, all these results are for regular problems and the term $\|u\|_{s+1}$ appears in the error bounds. By the regularity, we would have $\|u\|_{s+1} \approx \epsilon^{-s-1 / 2}$, which makes the error bounds meaningless for $\epsilon \ll 1$. Nevertheless, our earlier numerical experiments demonstrated $2 k+1$ order uniform superconvergence for flux at the nodal points under the Shishkin mesh for singularly perturbed problems in 1-D 14, which sparked the motivation for our investigation in this direction.

To end this introduction, we would like to point out that there are vast amounts of literature on numerical approximations for singularly perturbed problems; see, e.g., [6, 7, 8, 9, 10, 11] and the references therein. A central theme is uniform convergence.

\section{Fundamental INTERPolation PROPERTY FOR THE SINGULAR TERM}

When comparing singularly perturbed and non-singularly-perturbed problems, the main difficulty for singularly perturbed problems is the uniform approximation for the boundary layer term $q_{\epsilon}$. In this section, we define projection type interpolants and establish their approximation property for each of the aforementioned choices of $\tau$.

1) For $\tau=\tau_{\epsilon}$, we define $\pi_{\epsilon}^{ \pm}: C^{0} \rightarrow V_{N}^{\epsilon, k}$, such that

$$
\begin{gathered}
\int_{\Omega_{j}}\left(q_{\epsilon}-\pi_{\epsilon}^{ \pm} q_{\epsilon}\right) r=0, \quad \forall r \in \mathcal{P}_{k-1}\left(\Omega_{j}\right) ; \\
\left(q_{\epsilon}-\pi_{\epsilon}^{ \pm} q_{\epsilon}\right)_{j-1}^{ \pm}=0, \quad j=1, \ldots, N .
\end{gathered}
$$

We call $\pi_{\epsilon}^{-}\left(\pi_{\epsilon}^{+}\right)$the right (left) Radau interpolation operator.

2) For $\tau=\tau_{N}$, we define $\pi_{N}^{ \pm}: C^{0} \rightarrow V_{N}^{\epsilon, 1}\left(0,1-\tau_{N}\right) \cup V_{N}^{\epsilon, k}\left(1-\tau_{N}, 1\right)$, such that outside the boundary layer region,

$$
\int_{\Omega_{j}}\left(q_{\epsilon}-\pi_{N}^{ \pm} q_{\epsilon}\right)=0, \quad\left(q_{\epsilon}-\pi_{N}^{ \pm} q_{\epsilon}\right)_{j-1}^{ \pm}=0, \quad j=1, \ldots, N / 2,
$$

and $\pi_{N}^{ \pm}=\pi_{\epsilon}^{ \pm}$inside the boundary layer region. 
In order to simplify the notation, we discuss only $\pi_{N}=\pi_{N}^{+}$and $\pi_{\epsilon}=\pi_{\epsilon}^{+}$and all results are valid for $\pi^{-}$as well.

First, we define

$$
\bar{q}_{\epsilon, j}=\frac{1}{\left|\Omega_{j}\right|} \int_{\Omega_{j}} q_{\epsilon}
$$

Then, we can write explicitly for $x \in\left[x_{j-1}, x_{j}\right), j=1,2, \ldots, N / 2$ :

$$
\pi_{N} q_{\epsilon}(x)=q_{\epsilon}\left(x_{j-1}\right)+\frac{2}{H}\left(\bar{q}_{\epsilon, j}-q_{\epsilon}\left(x_{j-1}\right)\right)\left(x-x_{j-1}\right) .
$$

It is straightforward to verify that for $j=1,2, \ldots, N / 2$,

$$
\begin{gathered}
\left\|\pi_{N} q_{\epsilon}\right\|_{L^{2}\left(\Omega_{j}\right)}^{2}=\frac{5 H}{6}\left(q_{\epsilon}\left(x_{j-1}\right)-\bar{q}_{\epsilon, j}\right)^{2}+\frac{H}{2}\left(q_{\epsilon}\left(x_{j-1}\right)^{2}+\bar{q}_{\epsilon, j}^{2}\right) \\
\left\|\pi_{N} q_{\epsilon}\right\|_{L^{1}\left(\Omega_{j}\right)}=H\left|q_{\epsilon}\left(x_{j-1}\right)-2 \bar{q}_{\epsilon, j}\right| .
\end{gathered}
$$

To estimate the approximation for the singular part, we note that

$$
e^{-b_{0} \tau / \epsilon}= \begin{cases}\epsilon^{k+1}, & \tau=\tau_{\epsilon} \\ N^{-2 k-1}, & \tau=\tau_{N}\end{cases}
$$

A direct calculation yields, for $r=1,2$,

$$
\begin{gathered}
\int_{0}^{1-\tau} e^{-r b_{0}(1-x) / \epsilon} d x \leq \frac{\epsilon}{r b_{0}} \begin{cases}\epsilon^{r(k+1)}, & \tau=\tau_{\epsilon} \\
N^{-r(2 k+1)}, & \tau=\tau_{N}\end{cases} \\
\int_{1-\tau}^{1} e^{-r b_{0}(1-x) / \epsilon} d x \leq \frac{\epsilon}{r b_{0}}
\end{gathered}
$$

Then by the regularity assumption (1.3), we have

$$
\begin{gathered}
\left|q_{\epsilon}\right|_{W_{r}^{k+1}\left(0,1-\tau_{\epsilon}\right)}^{r} \leq C \epsilon^{-r(k+1)} \int_{0}^{1-\tau_{\epsilon}} e^{-r b_{0}(1-x) / \epsilon} d x \leq \frac{C \epsilon}{r b_{0}}, \\
\left\|q_{\epsilon}\right\|_{L^{r}\left(0,1-\tau_{N}\right)}^{r} \leq C \int_{0}^{1-\tau_{N}} e^{-r b_{0}(1-x) / \epsilon} d x \leq \frac{C \epsilon}{r b_{0}} N^{-r(2 k+1)}, \\
\left|q_{\epsilon}\right|_{W_{r}^{k+1}(1-\tau, 1)}^{r} \leq C \epsilon^{-r(k+1)} \int_{1-\tau}^{1} e^{-r b_{0}(1-x) / \epsilon} d x \leq \frac{C}{r b_{0}} \epsilon^{-r(k+1)+1} .
\end{gathered}
$$

Lemma 2.1. Let $q_{\epsilon}$ satisfy the regularity assumption (1.3) and let $\pi_{N}$ be defined by (2.2). Then we have, for $r=1,2$,

$$
\left\|\pi_{N} q_{\epsilon}\right\|_{L^{r}\left(0,1-\tau_{N}\right)}^{r} \lesssim \frac{\epsilon}{N^{r(2 k+1)}}
$$

Proof. From (2.4), we have

$$
\left\|\pi_{N} q_{\epsilon}\right\|_{L^{r}\left(0,1-\tau_{N}\right)}^{r} \approx H \sum_{j=1}^{N / 2}\left(q_{\epsilon}^{r}\left(x_{j-1}\right)+\bar{q}_{\epsilon, j}^{r}\right) .
$$


By the regularity assumption (1.3),

$$
\begin{aligned}
H \sum_{j=1}^{N / 2} q_{\epsilon}^{r}\left(x_{j-1}\right) & <C \int_{0}^{1-\tau_{N}} e^{-r b_{0}(1-x) / \epsilon} d x<\frac{C \epsilon}{r b_{0}} N^{-r(2 k+1)} ; \\
H \sum_{j=1}^{N / 2} \bar{q}_{\epsilon, j}^{r} & <C H \sum_{j=1}^{N / 2}\left(\frac{1}{H} \int_{x_{j-1}}^{x_{j}} e^{-b_{0}(1-x) / \epsilon} d x\right)^{r} \\
& <C \sum_{j=1}^{N / 2} \int_{x_{j-1}}^{x_{j}} e^{-r b_{0}(1-x) / \epsilon} d x \\
& =C \int_{0}^{1-\tau_{N}} e^{-r b_{0}(1-x) / \epsilon} d x<\frac{C \epsilon}{r b_{0}} N^{-r(2 k+1)} .
\end{aligned}
$$

Substituting (2.13) and (2.14) into (2.12), we obtain (2.11).

Now we are ready to prove the main interpolation result.

Theorem 2.2. Let $q_{\epsilon}$ satisfy the regularity assumption (1.3), $\pi_{\epsilon}$ and $\pi_{N}$ be defined by (2.1) and (2.2), respectively. Then we have, for $r=1,2$,

$$
\begin{gathered}
\left\|q_{\epsilon}-\pi_{N} q_{\epsilon}\right\|_{L^{r}\left(0,1-\tau_{N}\right)} \lesssim \frac{\epsilon^{1 / r}}{N^{2 k+1}} \\
\left\|q_{\epsilon}-\pi_{N} q_{\epsilon}\right\|_{L^{r}\left(1-\tau_{N}, 1\right)} \lesssim \epsilon^{1 / r}\left(\frac{2 k+1}{b_{0}} \cdot \frac{\ln N}{N}\right)^{k+1} ; \\
\left\|q_{\epsilon}-\pi_{\epsilon} q_{\epsilon}\right\|_{L^{r}(0,1)} \lesssim \epsilon^{1 / r}\left(\frac{k+1}{b_{0}} \cdot \frac{\ln \epsilon^{-1}}{N}\right)^{k+1} .
\end{gathered}
$$

Proof. First, (2.15) follows directly from the triangle inequality, (2.9), (2.10), and Lemma 2.1.

Note that $q_{\epsilon}-\pi_{N} q_{\epsilon}=0$ if $q_{\epsilon}$ is a polynomial of degree no more than $k$. By the Bramble-Hilbert Lemma and (2.10),

$$
\left\|q_{\epsilon}-\pi_{N} q_{\epsilon}\right\|_{L^{r}\left(1-\tau_{N}, 1\right)} \leq C h^{k+1}\left|q_{\epsilon}\right|_{W_{r}^{k+1}\left(1-\tau_{N}, 1\right)} \leq C \epsilon^{1 / r}\left(\frac{h}{\epsilon}\right)^{k+1} .
$$

Then (2.16) follows by using

$$
\frac{h}{\epsilon}=\frac{2 k+1}{b_{0}} \cdot \frac{\ln N}{N}
$$

Next, applying (2.8) and (2.10),

$$
\begin{aligned}
\left\|q_{\epsilon}-\pi_{\epsilon} q_{\epsilon}\right\|_{L^{r}(0,1)}^{r} & =\left\|q_{\epsilon}-\pi_{\epsilon} q_{\epsilon}\right\|_{L^{r}\left(0,1-\tau_{\epsilon}\right)}^{r}+\left\|q_{\epsilon}-\pi_{\epsilon} q_{\epsilon}\right\|_{L^{r}\left(1-\tau_{\epsilon}, 1\right)}^{r} \\
& \leq C H^{r(k+1)}\left|q_{\epsilon}\right|_{W_{r}^{k+1}\left(0,1-\tau_{\epsilon}\right)}^{r}+C h^{r(k+1)}\left|q_{\epsilon}\right|_{W_{r}^{k+1}\left(1-\tau_{\epsilon}, 1\right)}^{r} \\
& \leq C \epsilon\left(N^{-r(k+1)}+\left(\frac{h}{\epsilon}\right)^{r(k+1)}\right) \\
& \leq C \epsilon\left(\frac{k+1}{b_{0}} \cdot \frac{\ln \epsilon^{-1}}{N}\right)^{r(k+1)} .
\end{aligned}
$$

Note that in this case,

$$
\frac{h}{\epsilon}=\frac{k+1}{b_{0}} \cdot \frac{\ln \epsilon^{-1}}{N}
$$


Finally, 2.17) follows by taking the $r$-roots of (2.18).

\section{Proof of the main Result}

We denote $a(\cdot, \cdot)=a_{1}(\cdot, \cdot)$. Note that the scheme is consistent, i.e., the exact solution $q$ satisfies (1.10) and (1.11), and we have the orthogonality property

$$
a_{n}(q-Q, v)=0, \quad \forall v \in V_{N}^{\epsilon, k} .
$$

To establish the stability of the scheme, we set $v=Q$ in both (1.10) and (1.11) to obtain

$$
\begin{aligned}
a_{n}(Q, Q) & =\int_{x_{n-1}}^{1} Q Q^{\prime}+\epsilon^{-1} \int_{x_{n-1}}^{1} b Q^{2}+\left(Q_{n-1}^{+}\right)^{2}-\left(Q_{N}^{+}\right)^{2}+\sum_{j=n}^{N-1} Q_{j}^{+}[Q]_{j} \\
& =\int_{x_{n-1}}^{1} \epsilon^{-1} f Q ; \\
a_{n}(Q, Q) & =-\int_{x_{n-1}} Q^{\prime} Q+\epsilon^{-1} \int_{x_{n-1}}^{1} b Q^{2}-\sum_{j=n}^{N-1}[Q]_{j} Q_{j}^{-}=\int_{x_{n-1}}^{1} \epsilon^{-1} f Q .
\end{aligned}
$$

Combining (3.2) and (3.3), we have the following stability result:

$$
a_{n}(Q, Q)=\epsilon^{-1} \int_{x_{n-1}}^{1} b Q^{2}+\frac{1}{2} \sum_{j=n}^{N-1}[Q]_{j}^{2}+\frac{1}{2}\left[\left(Q_{n-1}^{+}\right)^{2}-\left(Q_{N}^{+}\right)^{2}\right]=\int_{x_{n-1}}^{1} \epsilon^{-1} f Q .
$$

By the orthogonality property, we have

$$
a_{n}\left(Q-q_{I}, Q-q_{I}\right)=a_{n}\left(q-q_{I}, Q-q_{I}\right),
$$

for any interpolant $q_{I} \in V_{N}^{\epsilon, k}$.

Letting $v=Q-q_{I}$ satisfy $\left(q-q_{I}\right)_{j}^{+}=0$, we obtain, by (1.10),

$$
b_{0}\|v\|^{2} \leq \epsilon a(v, v)=\epsilon a\left(q-q_{I}, v\right)=\epsilon\left(\int_{0}^{1-\tau}+\int_{1-\tau}^{1}\right)\left(q-q_{I}\right)\left(v^{\prime}+b \epsilon^{-1} v\right) .
$$

Recall that $b \geq b_{0}>0$.

Based on (1.3), we write

$$
q-q_{I}=\bar{q}-\bar{q}_{I}+q_{\epsilon}-q_{\epsilon, I} .
$$

The estimate for the regular part is standard:

$$
\begin{gathered}
\epsilon a\left(\bar{q}-\bar{q}_{I}, v\right) \leq C^{\prime}\left(\epsilon N^{-k}+N^{-k-1}\right)|\bar{q}|_{k+1}\|v\| \leq C\left(\epsilon N^{-k}+N^{-k-1}\right)\|v\|, \\
\left\|\bar{q}-\bar{q}_{I}\right\| \leq C N^{-k-1} .
\end{gathered}
$$

Proof of (1.12). For $\tau=\tau_{\epsilon}$, we set $q_{\epsilon, I}=\pi_{\epsilon}^{+} q_{\epsilon}$. By (3.5) and the inverse inequality,

$$
\left\|v^{\prime}\right\|_{(0,1-\tau)} \leq C H^{-1}\|v\|_{(0,1-\tau)}, \quad\left\|v^{\prime}\right\|_{(1-\tau, 1)} \leq C h^{-1}\|v\|_{(1-\tau, 1)},
$$

we have

$$
\begin{aligned}
\epsilon a\left(q_{\epsilon}-q_{\epsilon, I}, v\right) \leq & C\left(\epsilon H^{k}+H^{k+1}\right)\left|q_{\epsilon}\right|_{k+1,(0,1-\tau)}\|v\|_{(0,1-\tau)} \\
& +C\left(\epsilon h^{k}+h^{k+1}\right)\left|q_{\epsilon}\right|_{k+1,(1-\tau, 1)}\|v\|_{(1-\tau, 1)} \\
\leq & C \sqrt{\epsilon}\left(\frac{\ln \epsilon^{-1}}{N}\right)^{k}\|v\|,
\end{aligned}
$$


by (2.8) and (2.10). Applying (3.6) and (3.8) in (3.5) results in

$$
b_{0}\|v\|^{2}=b_{0}\left\|Q-q_{I}\right\|^{2} \leq C\left(\sqrt{\epsilon}\left(\frac{\ln \epsilon^{-1}}{N}\right)^{k}+\frac{1}{N^{k+1}}\right)\|v\| .
$$

Canceling $\|v\|$ on both ends, we obtain

$$
\left\|Q-q_{I}\right\| \leq C\left(\sqrt{\epsilon}\left(\frac{\ln \epsilon^{-1}}{N}\right)^{k}+\frac{1}{N^{k+1}}\right) .
$$

Using (2.17) and (3.7), we obtain

$$
\|q-Q\| \leq\left\|q-q_{I}\right\|+\left\|q_{I}-Q\right\| \leq C\left(\sqrt{\epsilon}\left(\frac{\ln \epsilon^{-1}}{N}\right)^{k}+\frac{1}{N^{k+1}}\right) .
$$

Proof of (1.14). When $\tau=\tau_{N}$, we set $q_{\epsilon, I}=\pi_{N}^{+} q_{\epsilon}$. By Theorem 2.2 and the inverse inequality,

$$
\begin{aligned}
\epsilon \int_{0}^{1-\tau}\left(q_{\epsilon}-q_{\epsilon, I}\right)\left(v^{\prime}+b \epsilon^{-1} v\right) & \leq\left\|q_{\epsilon}-q_{\epsilon, I}\right\|_{\left(0,1-\tau_{N}\right)}\left(\epsilon\left\|v^{\prime}\right\|+\|v\|\right) \\
& \leq C \sqrt{\epsilon} N^{-2 k-1}(N \epsilon+1)\|v\| .
\end{aligned}
$$

On the other hand, with $2 h=\tau / N$, we have, by the inverse estimate $\left\|v^{\prime}\right\| \leq$ $C h^{-1}\|v\|$,

$\epsilon \int_{1-\tau}^{1}\left(q_{\epsilon}-q_{\epsilon, I}\right)\left(v^{\prime}+b \epsilon^{-1} v\right) \leq C\left(\epsilon h^{k}+h^{k+1}\right)\left|q_{\epsilon}\right|_{k+1,(1-\tau, 1)}\|v\| \leq C \sqrt{\epsilon}\left(\frac{\ln N}{N}\right)^{k}\|v\|$.

Combining (3.10) and (3.11) yields (3.8) for this case. The rest is similar as in the proof of (1.12).

Proof of (1.15). Let $u_{\epsilon}=e^{\int_{x}^{x_{n-1}} b / \epsilon}$ with $x_{n-1} \in[1-\tau, 1)$. Then $u_{\epsilon}$ satisfies

$$
u_{\epsilon}^{\prime}+b \epsilon^{-1} u_{\epsilon}=0, \quad u_{\epsilon}\left(x_{n-1}\right)=1 \text {. }
$$

Setting $v=u_{\epsilon}$ in

$$
a_{n}(q-Q, v)=\int_{x_{n-1}}^{1}(q-Q)\left(v^{\prime}+b \epsilon^{-1} v\right)+(q-Q)_{n-1}^{+} v_{n-1}^{+}+\sum_{j=n}^{N-1}(q-Q)_{j}^{+}[v]_{j},
$$

we have, by the orthogonality property,

$$
\begin{aligned}
(q-Q)_{n-1}^{+} & =a_{n}\left(q-Q, u_{\epsilon}\right)=a_{n}\left(q-Q, u_{\epsilon}-\pi_{N}^{-} u_{\epsilon}\right) \\
& =\int_{x_{n-1}}^{1}\left[-\left(q-q_{I}\right)^{\prime}+b \epsilon^{-1}(q-Q)\right]\left(u_{\epsilon}-\pi_{N}^{-} u_{\epsilon}\right) .
\end{aligned}
$$

Here $\pi_{N}^{-}$is the projection operator defined in Section 2, and we have used

$$
a_{n}(q-Q, v)=\int_{x_{n-1}}^{1}\left[-(q-Q)^{\prime}+b \epsilon^{-1}(q-Q)\right] v-\sum_{j=n}^{N-1}[q-Q]_{j} v_{j}^{-} .
$$

We already have, from a previous argument,

$$
\left|\int_{x_{n-1}}^{1}\left[-\left(q_{\epsilon}-q_{\epsilon, I}\right)^{\prime}+b \epsilon^{-1}\left(q_{\epsilon}-Q\right)\right] v\right| \leq C(k) \epsilon^{-1 / 2}\left(\frac{\ln N}{N}\right)^{k}\|v\| .
$$


By Theorem 2.2, we have

$$
\left\|u_{\epsilon}-\pi_{N}^{-} u_{\epsilon}\right\|_{\left(x_{n-1}, 1\right)} \leq C(k) \sqrt{\epsilon}\left(\frac{\ln N}{N}\right)^{k+1} .
$$

Therefore,

$$
\left|\int_{x_{n-1}}^{1}\left[-\left(q_{\epsilon}-q_{\epsilon, I}\right)^{\prime}+b \epsilon^{-1}\left(q_{\epsilon}-Q\right)\right]\left(u_{\epsilon}-\pi_{N}^{-} u_{\epsilon}\right)\right| \leq C(k)\left(\frac{\ln N}{N}\right)^{2 k+1} .
$$

When $q=q_{\epsilon}$, we apply (3.13) to the right-hand side of (3.12) to obtain

$$
\left|(q-Q)_{n-1}^{+}\right| \leq C(k)\left(\frac{\ln N}{N}\right)^{2 k+1}
$$

which is (1.15). The proof of (1.13) is similar.

\section{ACKNOWLEDGMENT}

The authors would like to thank Professor Fatih Celiker for his comments and careful proofreading of the manuscript. The authors would also like to thank the two referees for their constructive comments and remarks which helped improve the quality and readablility of the paper.

\section{REFERENCES}

[1] C. Chen, Structure Theory of Superconvergence of Finite Elements, Hunan Science and Technology Press (in Chinese), Changsha, 2001.

[2] F. Celiker and B. Cockburn, Superconvergence of the numerical traces of discontinuous Galerkin and hybridized methods for convection-diffusion problems in one space dimension, Math. Comp. 76 (2007), 67-96. MR2261012 (2008e:65225)

[3] B. Cockburn, M. Luskin, C.-W. Shu, and E. Süli, Enhanced accuracy by post-processing for finite element methods for hyperbolic equations, Math. Comp. 72 (2003), 577-606. MR.1954957 (2004g:65129)

[4] B. Cockburn and C.-W. Shu, The local discontinuous Galerkin method for time-dependent convection-diffusion systems, SIAM Journal on Numerical Analysis, vol. 35 (1998), pp. 24402463. MR.1655854 (99j:65163)

[5] J. Douglas, Jr. and T. Dupont, Galerkin approximations for the two point boundary problem using continuous, piecewise polynomial spaces, Numer. Math. 22-2 (1974), 99-109. MR.0362922 (50:15360)

[6] W. Hundsdorfer and J.G. Verwer, Numerical Solution of Time-dependent Advection-diffusionreaction Equations, Springer, 2003. MR2002152 (2004g:65001)

[7] V. D. Liseikin, Layer Resolving Grids and Transformations for Singular Perturbation Problems, VSP, Boston, 2001.

[8] J.M. Melenk, HP-Finite Element Methods for Singular Perturbations, Springer, Berlin, 2002. MR.1939620 (2003i:65108)

[9] J.J. Miller, E. O'Riordan, and G.I. Shishkin, Fitted Numerical Methods for Singular Perturbation Problems, World Scientific, Singapore, 1996. MR.1439750 (98c:65002)

[10] K.W. Morton, Numerical Solution of Convection-Diffusion Problems, Chapman and Hall, London, 1996. MR:1445295 (98b:65004)

[11] H.-G. Roos, M. Stynes, and L. Tobiska, Numerical Methods for Singularly Perturbed Differential Equations, Springer, Berlin, 1996. (2nd and extended Edition 2008) MR1477665 (99a:65134)

[12] V. Thomée, Galerkin Finite Element Methods for Parabolic Problems, Springer, Berlin, 1997. MR.1479170 (98m:65007)

[13] M.F. Wheeler, A Galerkin procedure for estimating the flux for two-point boundary value problems, SIAM J. Numer. Anal. 11, 764 (1974). MR0383764(52:4644) 
UNIFORM SUPERCONVERGENCE OF DISCONTINUOUS GALERKIN METHOD

[14] Z.Q. Xie and Z. Zhang, Superconvergence of DG method for one-dimensional singularly perturbed problems, J. Comp. Math. 25-2 (2007), 185-200. MR2302756 (2008f:65230)

[15] Z. Zhang, Finite element superconvergent approximation for one-dimensional singularly perturbed problems, Numerical Methods for Partial Differential Equations 18 (2002), 374-395. MR:1895005 (2003c:65063)

College of Mathematics and Computer Science, Hunan Normal University, People's Republic of China

Department of Mathematics, Wayne State University, Detroit, Michigan 48202 